\title{
Electromagnetic simulations of JET ICRF ITER-like antenna with TOPICA and SSWICH asymptotic codes
}

\author{
Alena Křivská ${ }^{1, *}$, Volodymyr Bobkov ${ }^{2}$, Laurent Colas $^{3}$, Pierre Dumortier ${ }^{1,4}$, Frédéric Durodié ${ }^{1}$, Ernesto Lerche ${ }^{1,4}$, Philippe \\ Jacquet $^{4}$, Jonathan Jacquot ${ }^{2}$, Christopher Klepper ${ }^{5}$, Daniele Milanesio ${ }^{6}$ and JET contributors ${ }^{7}$ \\ ${ }^{1}$ LPP-ERM/KMS, Royal Military Academy, 30 Avenue de la Renaissance B-1000, Brussels, Belgium \\ ${ }^{2}$ Max-Planck-Institut für Plasmaphysik, D-85748 Garching, Germany \\ ${ }^{3}$ IRFM CEA, Cadarache F-13108 Saint Paul lez Durance, France \\ ${ }^{4}$ CCFE, Culham Science Centre, Abingdon, Oxfordshire OX14 3DB, UK \\ ${ }^{5}$ Oak Ridge National Laboratory, Oak Ridge, Tennessee 37831-6169, USA \\ ${ }^{6}$ Politecnico di Torino, Corso Duca degli Abruzzi 24, I-10129 Torino, Italy \\ ${ }^{7}$ See the author list of "Overview of the JET results in support to ITER" by X. Litaudon et al. to be published in Nuclear Fusion Special issue: \\ overview and summary reports from the 26th Fusion Energy Conference (Kyoto, Japan, 17-22 October 2016)
}

\begin{abstract}
Multi-megawatt Ion Cyclotron Range of Frequencies (ICRF) heating is routinely used in the JET tokamak. To increase the ICRF heating power available from the A2 antennas, the ICRF ITER-Like Antenna (ILA) was reinstalled for the 2015 JET ITER-like wall experimental campaign. The application of high levels of ICRF power typically results in increased plasma wall interaction which leads to the observation of enhanced influx of metallic impurities in the plasma edge. It is assumed that the impurity production is mainly driven by the parallel component of the Radio-Frequency (RF) antenna electric near-field, $\mathrm{E}_{/ /}$(parallel to the confinement magnetic field of the tokamak), that is rectified in a thin boundary layer (RF sheath). Torino Polytechnic Ion Cyclotron Antenna (TOPICA) code was used to compute $\mathrm{E}_{/ /}$field maps in front of the ILA and between its poloidal limiters in the presence of plasma using measured density profiles and various antenna feedings. $\mathrm{E}_{/ /}$field maps calculated between the poloidal limiters were used to estimate the poloidal distribution of RF-sheath Direct Current (DC) potential in this private region of the ILA and make relative comparison of various antenna electrical settings. For this purpose we used the asymptotic version of the Self-consistent Sheaths and Waves for Ion Cyclotron Heating Slow-Wave (SSWICH-SW) code. These estimations can help to study the formation of RF sheaths around the antenna and even at distant locations ( $\sim 3 \mathrm{~m}$ away).
\end{abstract}

\section{Introduction}

Multi-megawatt Ion Cyclotron Range of Frequencies (ICRF) heating with four A2 antennae is routinely used in the JET tokamak. To increase the ICRF heating power available from the A2 antennas, the ICRF ITER-Like Antenna (ILA) was reinstalled for the 2015 JET experimental campaign [1] with ITER-like wall (ILW). It uses compact strap array which has successfully been operated with power densities up to $6.2 \mathrm{MW} / \mathrm{m}^{2}$ on L-mode and $4.1 \mathrm{MW} / \mathrm{m}^{2}$ on $\mathrm{H}$-mode and voltages up to $42 \mathrm{kV}$ [2]. A detailed description of the ILA can be found in [3].

Application of high levels of ICRF power typically results in increased plasma wall interaction which leads to observation of enhanced influx of metallic impurities in the plasma edge [4]. Nevertheless the amount of the metallic impurities in JET plasmas is small and within tolerable limits without significantly affecting the plasma performance [5]. Previous studies (with the carbon fibre reinforced carbon wall) indicate that the RF-induced nickel influx per MW of ICRF power applied are comparable for the A2 and the ILA antennas [5], despite the higher power density (smaller radiating surface) of the latter. With the ILW, the ILA operation with only bottom half array powered leads to improvement of around $20 \%$ in terms of lower radiated power from the bulk plasma and the RFinduced tungsten content compared to A2 antennas operations. It is not yet clear from where in the machine the dominant tungsten contribution comes from [4]. Enhanced beryllium optical emission was also measured at some A2 antenna limiters that are magnetically connected to ILA [6, 7].

It is assumed that the impurity production is mainly driven by parallel component of Radio-Frequency (RF) antenna electric near-field $\mathrm{E}_{/ /}$(parallel to confinement magnetic field of the tokamak $\mathbf{B}_{0}$ ) that is rectified in thin boundary layers (RF sheaths). $\mathrm{E}_{/ /}$fields generate oscillating voltages, $\mathrm{V}_{\mathrm{RF}}$, across the rectifying RF-sheaths on $\mathbf{B}_{0}$ lines intercepting conducting obstacles. In these sheaths, the oscillating voltages $\mathrm{V}_{\mathrm{RF}}$ cause enhanced Direct Current (DC) plasma potentials $\mathrm{V}_{\mathrm{DC}}$. $\mathrm{RF}$ sheaths can develop over distances ranging from a few centimetres up to several 
meters away from the ICRF antennas, and cause plasma ion acceleration. The ions are accelerated along the confinement magnetic field lines, hit the obstacle and cause enhanced sputtering and localized heat flux. Advances in the field show that the induced currents on the passive antenna structures are the main source of $E_{/ /}$fields on the lateral faces of antenna side limiters [8].

In this contribution, Torino Polytechnic Ion Cyclotron Antenna (TOPICA) code [9] was used to compute $\mathrm{E}_{/ /}$field maps in front of the ILA and between its poloidal limiters for operation of only top, only bottom and full antenna array in the presence of plasma using experimentally measured plasma density profiles. $\mathrm{E}_{/ /}$field maps calculated between the poloidal limiters were used to estimate poloidal distribution of RF-sheath DC potential $V_{D C}$ in this private region of the ILA. For this purpose we used the asymptotic version of the Self-consistent Sheaths and Waves for Ion Cyclotron Heating Slow-Wave (SSWICH-SW) code with radiating boundary conditions [10]. These estimations can help to study the formation of RF sheaths around the antenna and even at distant locations ( $\sim 3 \mathrm{~m}$ away) and make association to some variations of the Be line emission that were measured spectroscopically on some of the A2 antenna limiters when the magnetic connection from observation point to the active ILA was moved via a $\mathrm{q}_{95}$ scan [7].

\section{Description of the simulations}

$\mathrm{E}_{/ /}$field maps were computed with TOPICA code. To perform simulation with the TOPICA code, the 3D curved model of ILA with limiters was placed into a recess and limited by an aperture. The aperture follows the curvature of the antenna structure and represents the interface between the antenna region, which is in vacuum, and the plasma region. The vacuum-plasma interface was placed $1 \mathrm{~cm}$ in front of the leading edge of the poloidal limiters as shown in Figure 1. First surface where we calculated the $\mathrm{E}_{/ /}$field was defined in the vacuum region $5 \mathrm{~mm}$ behind the vacuumplasma interface. It follows curvature of the antenna structure too. Second surface for the $\mathrm{E}_{/ /}$field calculations was placed between the poloidal limiters, $4 \mathrm{~mm}$ behind their leading edge as shown in Figure 1. $\mathrm{E}_{/ /}$maps from the second surface were used as input for estimation of $\mathrm{V}_{\mathrm{DC}}$ between the poloidal limiters.
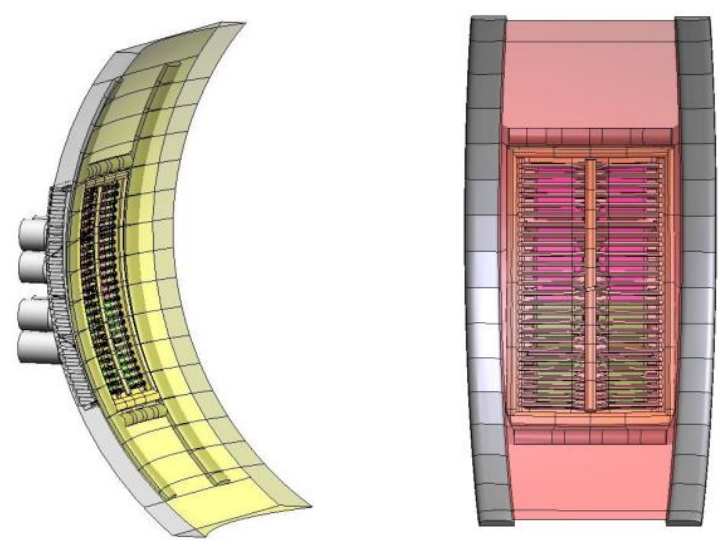

Fig. 1. ILA model with the vacuum plasma interface (on the left) and surface for $\mathrm{E}_{/ /}$field calculations between the poloidal limiters (on the right).

For the $E_{/ /}$field maps computations we used as input plasma density and temperature profiles from L-mode shot \#90454 that were measured with lithium beam emission spectroscopy system. During the shot $\mathrm{q}_{95}$ (safety factor at $95 \%$ of the plasma minor radius) varied from 3.04 to 3.92 (Figure 2), Radial Outer Gap (ROG) was equal to $6 \mathrm{~cm}$, central plasma density was around $3 \cdot \mathrm{e}^{19} \mathrm{~m}^{-3}$ and central plasma temperature was equal to $1.85 \mathrm{keV}$. Central static magnetic field was $2.375 \mathrm{~T}, 10^{\circ}$ tilted with respect to the toroidal direction. Plasma geometry was assumed slab with temperature and density gradients along minor radius of the tokamak chamber. Simulations were done for frequency 42 $\mathrm{MHz}$ and minority heating scheme with $3.5 \%$ of hydrogen and $96.5 \%$ of deuterium. Different antenna feeding was adopted: a) full array $(0 \pi)$, b) full array $(0-\pi / 2)$, c) only top half array $(0 \pi)$ and $d)$ only bottom half array $(0 \pi)$.

$\mathrm{E}_{/ /}$field maps in front of the antenna were also used for work that studies the RF sheath enhanced plasma surface interactions using optical emission spectroscopy on JET with ILW [7]. For that investigation we used the same antenna model and plasma settings as mentioned in the previous text. $\mathrm{E}_{/ /}$field maps were calculated on the first surface that was located $5 \mathrm{~mm}$ in front of the leading edge of the poloidal limiters. In that case simulations were performed for only top half array $(0-\pi / 2)$ and only bottom half array $(0-\pi / 2)$ antenna feedings.

For estimation of the poloidal distribution of the rectified DC plasma potential $V_{D C}$ between the poloidal limiters of the ILA we used the asymptotic version of the SSWICH-SW code with radiating boundary conditions. It was chosen because it is rapid, robust, always provides systematically one solution for each set of input parameters and convergence is guaranteed. It is suitable for relative comparisons of antenna designs and electrical settings. Principal disadvantages of the code are related to number of approximations made on the original physical model. Parallel DC plasma conductivity is taken as infinite while transverse plasma conductivity is neglected in the simplified model of DC plasma biasing. The potential $\mathrm{V}_{\mathrm{DC}}$ is homogeneous all along the open field lines and depends only on the voltages $\mathrm{V}_{\mathrm{RF}}$ at both ends of these field lines. Main input to the SSWICH-SW code is the $\mathrm{E}_{/ /}$field map evaluated between the poloidal limiters. Simple 3dimensional parallelepiped simulation domain with walls normal to the straight magnetic field lines and only slow wave, responsible for excitation of the RF sheaths when the side walls are normal to $\mathbf{B}_{0}$, were only adopted. Sketch of the simulation domain can be found in [11]. 


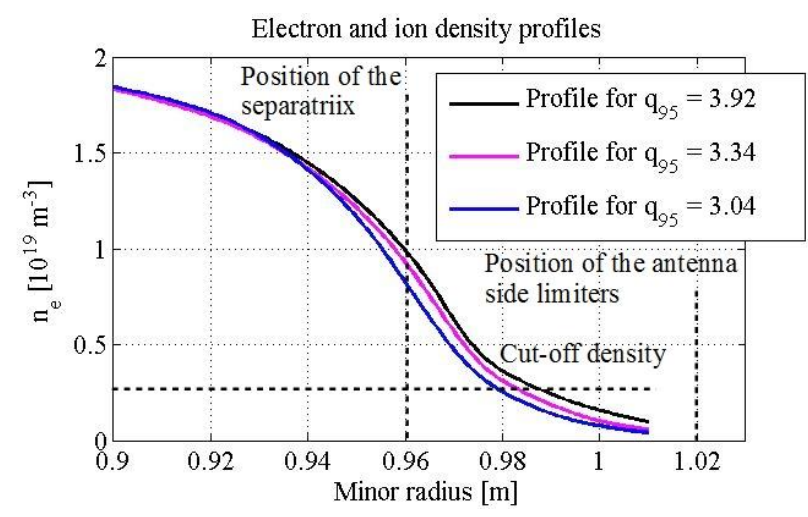

Fig. 2. Electron and ion density profiles with varying $\mathrm{q}_{95}$ that were measured during shot \#90454 with Li beam emission spectroscopy.

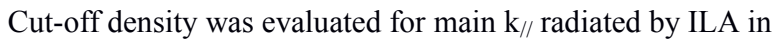
dipole phasing.

\section{Simulation results}

\subsection{Estimation of spatial distribution of $E_{/ /}$near field}

$\mathrm{E}_{/ /}$field maps calculated $5 \mathrm{~mm}$ in front of the leading edge of the poloidal limiters for various antenna feedings are shown in Figures 3-5. These maps were obtained for the profile with $\mathrm{q}_{95}=3.34$. Results were normalized to $450 \mathrm{~kW}$ of coupled power to the target plasma.

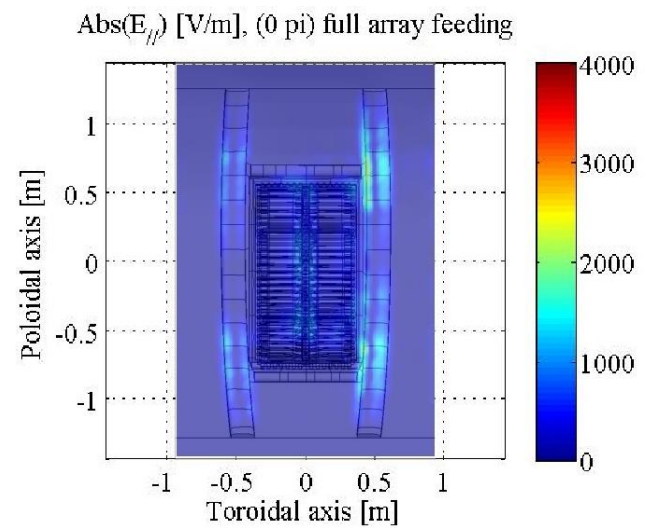

Fig. 3. Absolute values of $E_{/ /}$field for $(0 \pi)$ full array feeding.

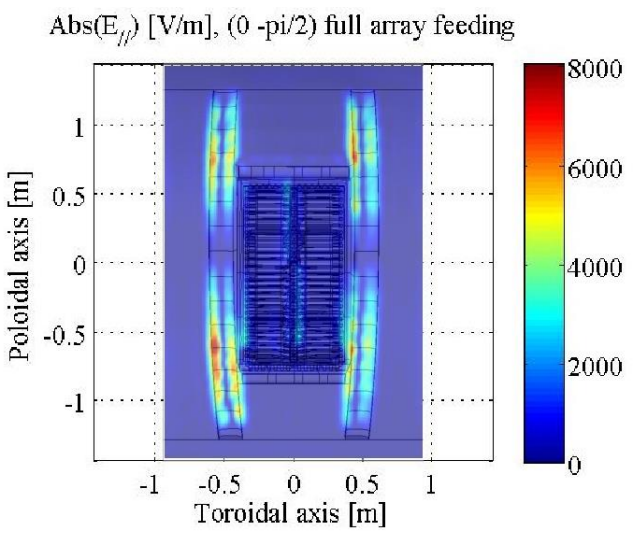

Fig. 4. Absolute values of $E_{/ /}$field for $(0-\pi / 2)$ full array feeding. Used scale is not the same as in case of the other $\mathrm{E}_{/ /}$field maps.

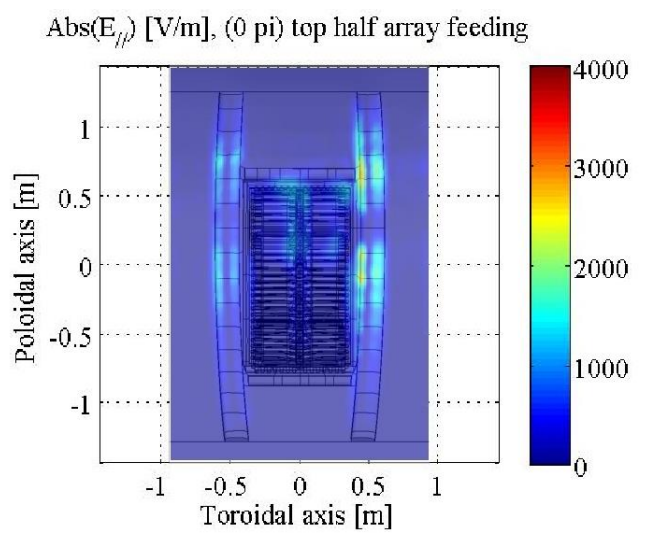

Fig. 5. Absolute values of $E_{/ /}$field for $(0 \pi)$ only top half array feeding.

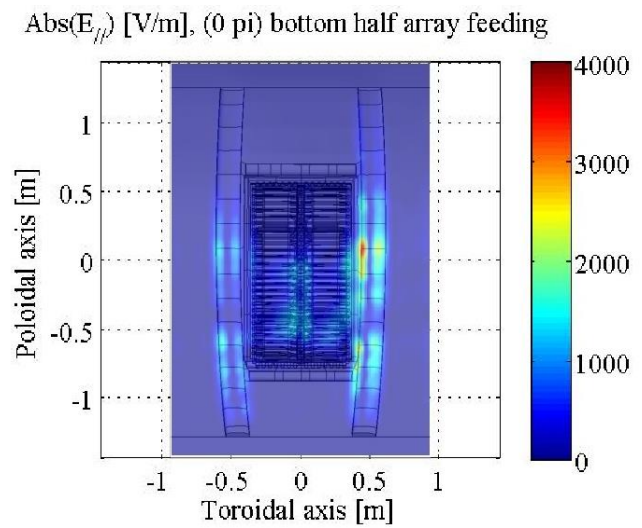

Fig. 6. Absolute values of $E_{/ /}$field for $(0 \pi)$ only bottom half array feeding.

Areas with peak values of the $E_{/ /}$field are in the corners of the maps along the poloidal limiters. For full array $(0 \pi)$ feeding the increased field values are in poloidal direction from $\mathrm{y}=-0.8 \mathrm{~m}$ to $\mathrm{y}=-0.6 \mathrm{~m}$ and from $\mathrm{y}=0.4 \mathrm{~m}$ to $\mathrm{y}=0.8$ $\mathrm{m}$. For full array $(0-\pi / 2)$ feeding it is from $\mathrm{y}=-1.2 \mathrm{~m}$ to $\mathrm{y}=$ $-0.2 \mathrm{~m}$ and from $\mathrm{y}=0.3 \mathrm{~m}$ to $\mathrm{y}=1.2 \mathrm{~m}$. In case of only top half array $(0 \pi)$ feeding the increased field values are from from $\mathrm{y}=-0.3 \mathrm{~m}$ to $\mathrm{y}=0.15 \mathrm{~m}$ and from $\mathrm{y}=0.4 \mathrm{~m}$ to $\mathrm{y}=1$ $\mathrm{m}$. In case of only bottom half array $(0 \pi)$ feeding it is from $\mathrm{y}=-0.9 \mathrm{~m}$ to $\mathrm{y}=-0.6 \mathrm{~m}$ and from $\mathrm{y}=-0.35 \mathrm{~m}$ to $\mathrm{y}=0.45 \mathrm{~m}$.

Highest $E_{/ /}$field values were obtained for $(0-\pi / 2)$ full array antenna feeding. For this setting there is low wave number parallel to the magnetic field $\mathrm{k}_{/ /}$dominant in the antenna spectrum. This ensures good coupling to plasma but absorption of the waves in the plasma is smaller and larger fraction of the RF power can excite coaxial and surface modes. Peak $E_{/ /}$field values obtained for half-array feedings are higher than for $(0 \pi)$ full array feeding. This is due to higher power density for half-array feedings compared to full array feeding for constant coupled power. Main source of the increased values of $E_{/ /}$fields are image current densities that circulate on the lateral faces of the antenna side limiters.

\subsection{Estimation of distribution of RF-sheath DC potential}


$\mathrm{E}_{/ /}$field maps calculated in absence of sheaths on surface between the poloidal limiters, $4 \mathrm{~mm}$ behind their leading edge, were used as main input to the SSWICH-SW code. This location was chosen to include properly influence of the current density circulation on the poloidal limiters. Calculated $\mathrm{V}_{\mathrm{DC}}$ potentials were averaged in radial direction in area between the leading edge of the polodal limiters and position $4 \mathrm{~mm}$ behind their leading edge. Resulting distributions in poloidal direction for various antenna feedings are shown in Figures 7-10. They were normalized to $450 \mathrm{~kW}$ of coupled power to the target plasma. Poloidal distributions of peak values of $\mathrm{V}_{\mathrm{DC}}$ potential correspond to the poloidal distributions of increased values of $E_{/ /}$field. It is due correlation of $V_{D C}$ potentials with amplitudes of $E_{/ /}$ fields at the lateral sides of the input field maps. More investigations about this effect were performed in $[11,12]$.

For full array $\left(\begin{array}{ll}0 & \pi\end{array}\right)$ feeding the peak values of $\mathrm{V}_{\mathrm{DC}}$ potentials are in poloidal direction around $\mathrm{y}=-0.7 \mathrm{~m}$ and $\mathrm{y}$ $=0.65 \mathrm{~m}$. For full array $(0-\pi / 2)$ feeding it is around $\mathrm{y}=$ $0.7 \mathrm{~m}, \mathrm{y}=0.8 \mathrm{~m}$ and $\mathrm{y}=0.95 \mathrm{~m}$. In case of only top half array $(0 \pi)$ feeding the peak values of $\mathrm{V}_{\mathrm{DC}}$ potentials are around $y=-0.05 \mathrm{~m}$ and $\mathrm{y}=0.65 \mathrm{~m}$. In case of only bottom half array $(0 \pi)$ feeding it is around $y=-0.7 \mathrm{~m}$ and $\mathrm{y}=0.07$ $\mathrm{m}$. Highest $\mathrm{V}_{\mathrm{DC}}$ potentials values were obtained for $(0-\pi / 2)$ full array antenna feeding. Higher $\mathrm{V}_{\mathrm{DC}}$ potentials values were otained for profiles with lower $\mathrm{q}_{95}$.

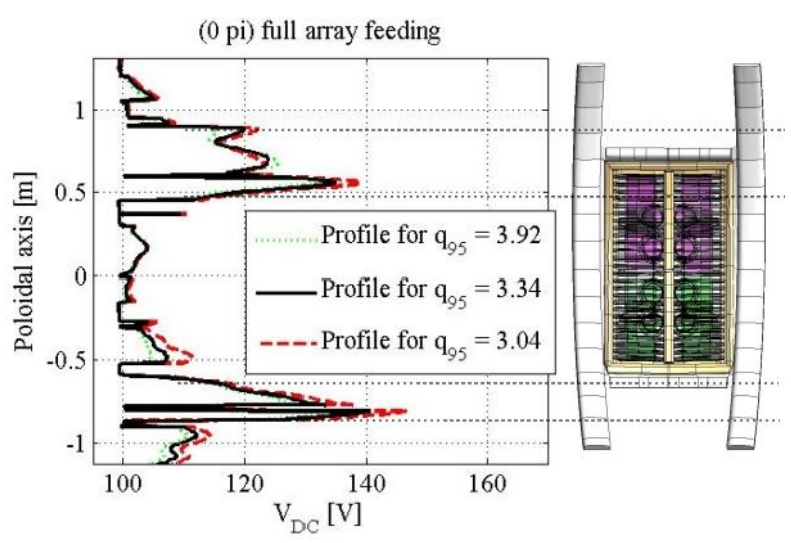

Fig. 7. Distribution of $V_{D C}$ potential for $(0 \pi)$ full array feeding.

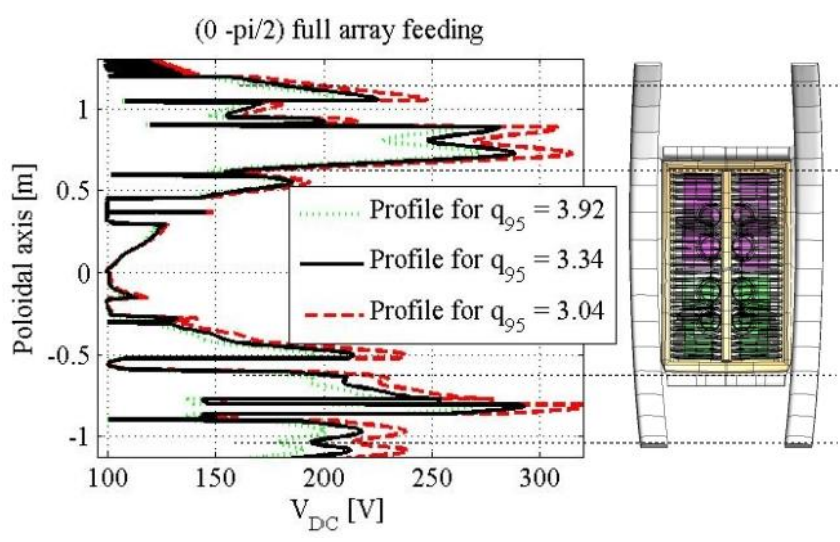

Fig. 8. Distribution of $V_{D C}$ potential for $(0-\pi / 2)$ full array feeding. Used scale is not the same as in case of the other potential plots.

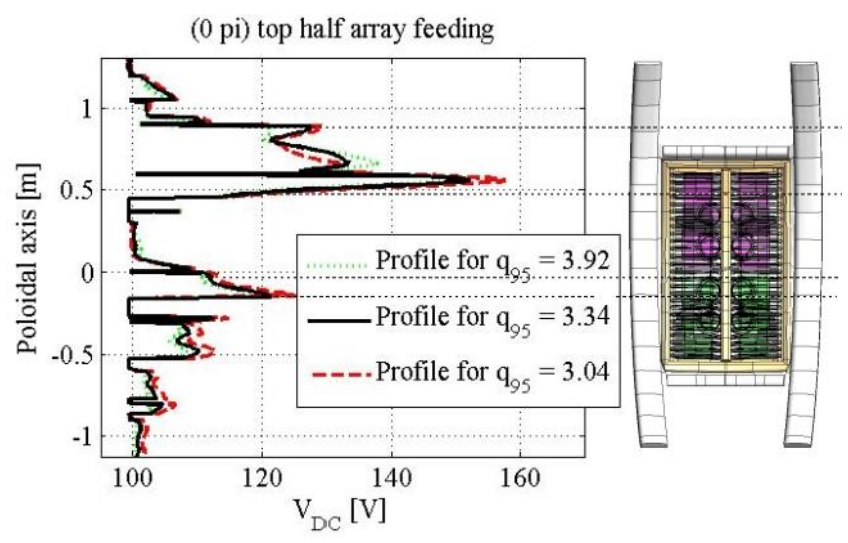

Fig. 9. Distribution of $V_{D C}$ potential for $(0 \pi)$ top half feeding.

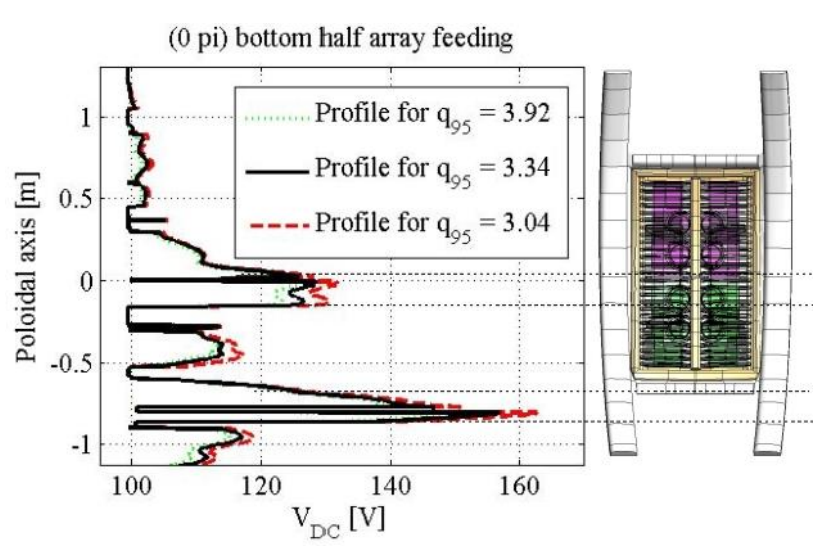

Fig. 10. Distribution of $V_{D C}$ potential for $(0 \pi)$ bottom half feeding.

\section{Conclusions and prospects}

Distributions of $\mathrm{E}_{/ /}$field and $\mathrm{V}_{\mathrm{DC}}$ potential were calculated and areas with increased values were identified. Relative comparison for various antenna feedings was performed. Main source of the increased values of $E_{/ /}$field and $V_{D C}$ potential was identified as image current densities that circulate on the poloidal antenna limiters. Higher $\mathrm{V}_{\mathrm{DC}}$ potentials values were computed for $(0-\pi / 2)$ phasing and for profiles with lower $\mathrm{q}_{95}$.

In the next step, we will investigate how distribution of $E_{/ /}$field and $V_{D C}$ potential changes with changing frequency, ROG and plasma parameters. Further, heat loads will be estimated using the $\mathrm{V}_{\mathrm{DC}}$ potential values. The near-field and $\mathrm{RF}$ sheath models should be validated using experimenal data. Comparison of calculated near fields and $V_{D C}$ potentials will be performed for the A2 antenna and the ILA.

Acknowledgements. This work has been carried out within the framework of the EUROfusion Consortium and has received funding from the European research and training programme under grant agreement $\mathrm{N}^{\circ}$ 633053. The views and opinions expressed herein do not necessarily reflect those of the European Commission. This work has been carried out using Cineca's supercomputer system Marconi. 


\section{References}

1. P. Dumortier et al., submitted to Fusion Eng. Des.

2. F. Durodié et al., Plasma Phys. Control. Fusion 54, 7 (2012)

3. F. Durodié el al., Fusion Eng. Des. 74, 223-228 (2005)

4. V. Bobkov et al., Nuclear Materials and Energy, in press (2016)

5. A. Czarnecka et al., Plasma Phys. Control. Fusion 54, (2012) 074013 (13pp)

6. P. Jacquet et al., submitted to Eur. Phys. J.

7. C. C. Klepper et al., submitted to Eur. Phys. J.

8. L. Colas et al., Nucl. Fus. 45, 767 (2005)

9. V. Lancellotti et al., Nucl. Fus. 46, S476 (2006)

10. L. Colas et al., Phys. Plasmas 19, 092505 (2012)

11. L. Colas et al., Plasma Phys. Control. Fusion 59, (2012)

12. A. Křivská et al., AIP Conf. Proc. 1689, 050002 (2015) 